\title{
A Theoretical Argument Why the t-Copula Explains Credit Risk Contagion Better than the Gaussian Copula
}

\author{
Didier Cossin, ${ }^{1,2,3}$ Henry Schellhorn, 1, 2, 3 Nan Song, 1, 2,3 \\ and Satjaporn Tungsong $1,2,3$
}

${ }^{1}$ IMD, 1001 Lausanne, Switzerland

${ }^{2}$ Claremont Graduate University, Claremont, CA 91711, USA

${ }^{3}$ Thammasat University, Bangkok, Thailand

Correspondence should be addressed to Henry Schellhorn, henry.schellhorn@cgu.edu

Received 19 December 2009; Accepted 23 February 2010

Academic Editor: Chin Lai

Copyright (C) 2010 Didier Cossin et al. This is an open access article distributed under the Creative Commons Attribution License, which permits unrestricted use, distribution, and reproduction in any medium, provided the original work is properly cited.

One of the key questions in credit dependence modelling is the specfication of the copula function linking the marginals of default variables. Copulae functions are important because they allow to decouple statistical inference into two parts: inference of the marginals and inference of the dependence. This is particularly important in the area of credit risk where information on dependence is scant. Whereas the techniques to estimate the parameters of the copula function seem to be fairly well established, the choice of the copula function is still an open problem. We find out by simulation that the t-copula naturally arises from a structural model of credit risk, proposed by Cossin and Schellhorn (2007). If revenues are linked by a Gaussian copula, we demonstrate that the t-copula provides a better fit to simulations than does a Gaussian copula. This is done under various specfications of the marginals and various configurations of the network. Beyond its quantitative importance, this result is qualitatively intriguing. Student's t-copulae induce fatter (joint) tails than Gaussian copulae ceteris paribus. On the other hand observed credit spreads have generally fatter joint tails than the ones implied by the Gaussian distribution. We thus provide a new statistical explanation why (i) credit spreads have fat joint tails, and (ii) financial crises are amplified by network effects.

\section{Introduction}

One of the key questions in credit dependence modelling is currently the specification of the copula function linking the marginals of default variables. Several books have been written on copulae as well as their application to finance, for example, Cherubini et al. [1], Embrechts et al. [2], Joe [3], and Nelsen [4]. We refer to these books for an exposition of copula theory. The main application of copulae seems to be the following. In several domains, 
and in particular in credit risk, there is often a lack of information to model the dependence between random variables. However, there is sufficient information to model the marginals of each random variable. It is in this particular context that-copulae functions are important for dependence modelling. The estimation of the joint distribution can thus be decoupled into estimation of the marginals, which is more robust, and estimation of the copula function. This estimation method, coupled with maximum likelihood (MLE) estimation, is often called inference for the margins, or IFM (see, e.g., Cherubini et al. [1, Section 5.3]).

Whereas the techniques to estimate the parameters of the copula function seem to be fairly well established, the choice of the copula function is still an open problem. Among several copulae, researchers in finance seem to have employed mostly the Gaussian, $t$, and Archimedean (family of) copulae. One of the advantages of the Gaussian copula is ease of simulation, while the Archimedean copula offers some advantages for inference. There has been wide-spread use of Gaussian copulas by rating agencies lately.

The initial goal of this research was to help practitioners in the selection of a particular copula function. More specifically, our main goal was to compare t-copulae with the Gaussian copula as a way to model counterparty risk. We found out by simulation that the $t$-copula naturally arises from a structural model of credit risk, proposed by Cossin and Schellhorn [5], and henceforth abbreviated CS model. The CS model links operating revenue to the credit spreads of firms in a network economy. If revenues are linked by a Gaussian copula, we demonstrate that the $\mathrm{t}$-copula provides a better fit to simulations than does a Gaussian copula. This is done under various specifications of the marginals and various configurations of the network. Beyond its quantitative importance, this result is qualitatively intriguing. It has been recognized by various researchers (see, e.g., Bluhm [6]), that $t$-copulae induce fatter (joint $)^{1}$ tails than Gaussian copulae ceteris paribus. On the other hand, the finance literature has abundantly documented in the last twenty years that credit spreads have generally fatter joint tails than implied by the Gaussian distribution. We thus provide a new statistical explanation why credit spreads have fat joint tails: even if the driver of credit spreads (namely revenue) has normal tails, because of counterparty relationships, credit spreads have fatter joint tails. Thus, financial crises are amplified by network effects.

This work also confirms the plausibility of the CS model. Indeed, under the hypothesis that (i) the (CS) model is valid and (ii) revenue has normal tails, we conclude that credit spreads have fat (joint) tails. Since fat joint tails are empirically observed, this seems to indicate that the (CS) model does a good job at explaining contagion. In this article, we do not dwell on this positive feature of our model. Indeed most credit risk models attempt to explain nonnormal risk of contagion, and a model which does not exhibit this feature would not be very interesting.

The curious reader may wonder what is the point of doing a statistical inference on a deterministic model. It is tempting to allude to Einstein's criticism of quantum mechanics "God does not play with dice". While there may be a purely deterministic description of microphysical phenomena beyond quantum mechanics, the latter theory proved to be fruitful for many years. We could argue that the same applies in credit risk. While a better description of credit risk if probably is given by models like the (CS) model, the lack of identifiability of model parameters (due to lack of disclosure of exposure parameters $\lambda$ ) forces practitioners to resort to statistical models. The point is that the statistical model must be indistinguishable from benchmark deterministic models.

Our article is composed of three parts. We first expose the models of network economies that we test. We then describe the statistical methodology. The statistical methodology is split into two parts: parameter estimation and hypothesis testing. In the 


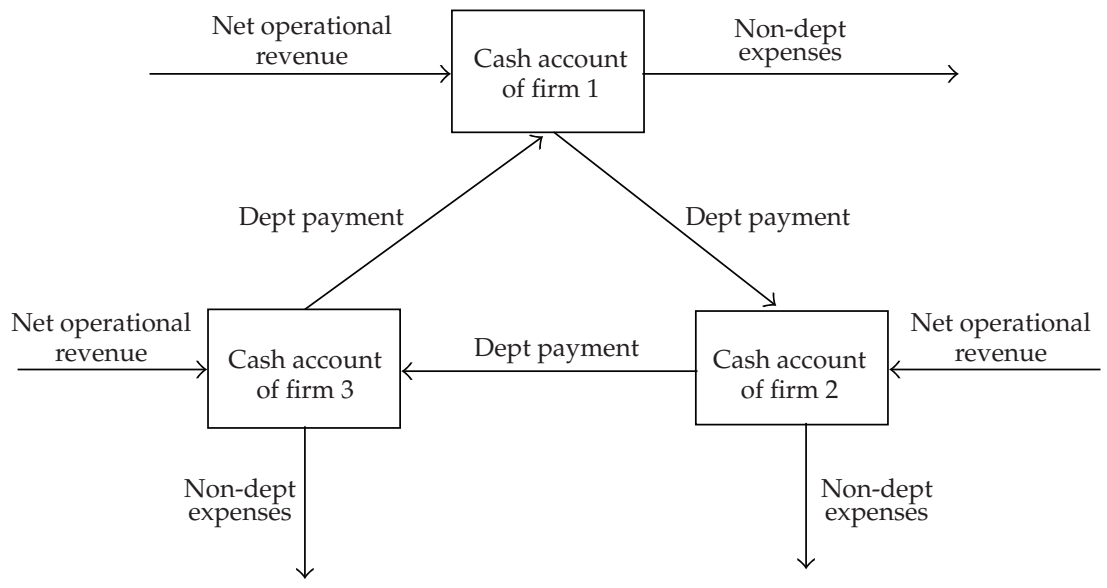

Figure 1: An example of a network economy with 3 firms.

hypothesis testing section, we compare the two null-hypotheses: Gaussian copula, and tcopula. Finally, we present the results.

\section{Models of Network Economies}

We first describe briefly the (CS) model in general and then introduce the types of network economies that we will study. We then discuss the distributional assumptions.

\subsection{The CS Model}

In the CS model, each firm is (potentially) at the same time a borrower from another (or several) firm(s) in the network and a lender to another (or several) firm(s). Our main assumption is not only that the quantity of debt is fixed, but also the network of lending and borrowing is fixed. In other terms, firms have preferred lenders, and the amount borrowed from them does not change with time. In addition to their borrowing and lending function, each firm produces goods and distributes dividends to its shareholders, in a manner similar to Leland's [7] model. We show an example of such a network economy in Figure 1.

We call $\bar{v}_{i}$ the long run operational revenue rate of firm $i$. In the CS model, this rate is not directly observable. At each time $t$ the market calculates an estimator $\widehat{v}_{i}(t)$ of $\bar{v}_{i}$. For the sake of brevity in this document, we will call $\widehat{v}_{i}$ simply the production revenue. The total revenue $\widehat{\alpha}_{i}$ of firm $i$ consists of its operational revenue plus debt payments from its borrowers. The main assumption of our model is that network relationships are fixed: debt payments from firm $k$ to firm $i$ are, at all times, proportional to the total revenue of firm $k$. In other terms, there are constants $\lambda_{k i}$ so that

$$
\widehat{\alpha}_{i}(t)=\widehat{v}_{i}(t)+\sum_{k} \widehat{\alpha}_{k}(t) \lambda_{k i}
$$

The payout ratio $\delta_{i}$ expresses what percentage of total expenses of firm $i$ is distributed to bondholders and equityholders. It is modelled as a geometric Brownian motion with relative 
drift $\mu_{i}$ and volatility $\sigma_{i}$. A payout ratio superior to one corresponds to a recapitalization of the firm. We assume that the payout ratio is independent of the level of expenses. Payout ratios across firms can be correlated. Upon default, firm $i$ incurs a loss rate of $w_{i}$. Theorem 2.1 was proved in Cossin and Schellhorn [5]:

Theorem 2.1. In steady state, the values of equity $S$ and debt $B$ are, for finite $N$, and $t$ not a bankruptcy time:

$$
\begin{aligned}
& S_{i}(t, \omega)=\widehat{\alpha}_{i}(t, \omega) s\left(\delta_{i}(t, \omega) ; \delta_{i}(0), \mu_{i}, \sigma_{i}, w_{i}, r\right)+O\left(\frac{1}{\sqrt{N}}\right), \\
& B_{i}(t, \omega)=\widehat{\alpha}_{i}(t, \omega) b\left(\delta_{i}(t, \omega) ; \delta_{i}(0), \mu_{i}, \sigma_{i}, w_{i}, r\right)+O\left(\frac{1}{\sqrt{N}}\right) .
\end{aligned}
$$

The parameter $N$ scales the production revenue intensity, as is common in diffusion approximations. We refer to Cossin and Schellhorn [5] for a full definition of $N$. Roughly speaking, a large $N$ corresponds to a large cash account, which acts as a (partial) buffer against cash flow risk. In this paper, we make the assumption that $N$ is very large, for simplicity. The functions $s$ and $b$ are fully analytical and equal (mutatis mutandis) to the formulae for the price of debt and equity in Leland's [7] model.

We want to show that the copula function of the counterparty risk premium (which we define in (2.8)) is equal to the copula function of total revenue. This occurs in the case of a stationary debt structure a la Leland and Toft [8], where the distribution of principal is uniform between $[t, t+T]$. In such an environment, a constant bankruptcy level is optimal. It can also be seen that liquidity risk is also decoupled from counterparty risk. More formally, let $B_{i}(t, T, \omega)$ be the total value of debt. There is now a new function $b^{\mathrm{LT}}$ so that

$$
B_{i}(t, T, \omega)=\widehat{\alpha}_{i}(t, \omega) b_{i}^{\mathrm{LT}}\left(\delta_{i}(t, \omega), T ; \delta_{i}(0), \mu_{i}, \sigma_{i}, w_{i}, r\right)+O\left(\frac{1}{\sqrt{N}}\right)
$$

Differentiating (2.3) with respect to $T$, we see that the value of debt with maturity $[T, T+d T]$ is equal to

$$
\frac{\partial}{\partial T} B_{i}(t, T, \omega) d T=\widehat{\alpha}_{i}(t, \omega) \frac{\partial}{\partial T} b_{i}^{\mathrm{LT}}\left(\delta_{i}(t, \omega), T ; \delta_{i}(0), \mu_{i}, \sigma_{i}, w_{i}, r\right) d T+O\left(\frac{1}{\sqrt{N}}\right) .
$$

On the other hand, we can rewrite the left-hand-side as

$$
\frac{\partial}{\partial T} B_{i}(t, T, \omega) d T \equiv \frac{L_{i}}{T} \exp \left(-\left(r+\mathrm{cs}_{i}(t, T)\right) T\right) d T,
$$

where $L_{i}$ is the total principal and $\mathrm{cs}_{i}(T)$ the credit spread of firm $i$ for maturity $T$. For simplicity we write

$$
\varphi_{i}(t, T, \omega)=\left.\frac{\partial b_{i}^{\mathrm{LT}}}{\partial T}\right|_{\delta_{i}(t, \omega), T}
$$


Thus, equating (2.4) with (2.5), and taking $N \rightarrow \infty$, we have

$$
r+\operatorname{cs}_{i}(t, T, \omega)=\frac{1}{T}\left[\ln \left(\frac{L_{i}}{\widehat{\alpha}_{i}(t, \omega) T}\right)-\ln \varphi_{i}(t, T, \omega)\right]
$$

Our main goal in this article is to assess the impact of counterparty risk. We are thus more interested in the credit risk coming from $\widehat{\alpha}_{i}$ than the credit risk coming from $\varphi_{i}$ in the last expression. We thus define the counterparty risk premium crp $p_{i}$ and a bankruptcy risk premium $\operatorname{brp}_{i}$ :

$$
\begin{gathered}
\operatorname{crp}_{i}=\frac{1}{T} \ln \left(\frac{L_{i}}{\widehat{\alpha}_{i}(t, \omega) T}\right), \\
\operatorname{brp}_{i}=-\frac{1}{T} \ln \varphi_{i}(t, T, \omega)-r .
\end{gathered}
$$

The counterparty risk premium stems from the network term $\widehat{\alpha}_{i}$ and the bankruptcy risk premium comes from the idiosyncratic variable $\delta_{i}$, which represents the payout ratio. While the bankruptcy risk premium is different for debt and for equity, the counterparty risk premium is the same for both securities. Assembling (2.8), (2.9), and (2.7), we see that we can decompose the credit spread into counterparty risk premium and bankruptcy risk premium:

$$
\operatorname{crp}_{i}+\operatorname{brp}_{i}=\mathrm{cs}_{i}
$$

It is well known that the copula function is unchanged under monotone transformations, such as the transformation (2.8) between total revenue $\widehat{\alpha}_{i}$ and counterparty risk premium $\operatorname{crp}_{i}$. We thus showed the following fact.

Fact. The copula function of the counterparty risk premium is the same as the copula function of total revenue.

Finally, we perform only a static analysis; that is, we analyze only the counterparty risk premium viewed as a random variable. What remains to be specified are then the network configurations and the distributional assumptions.

\subsection{Networks}

We analyze 3 different network configurations, that is possible relationships (networks) between the production revenue $\widehat{v}_{i}$ and the total revenue $\widehat{\alpha}_{i}$ :

(i) a "triangular", or "full" network,

(ii) a "star" network,

(iii) a "series" network. 
Table 1

(a)

\begin{tabular}{|c|c|c|c|}
\hline \multicolumn{2}{|c|}{ Full (Triangular) Network } & \multicolumn{2}{|c|}{$P$-value } \\
\hline No. of firms & No. of revenue scenarios & First K-S Test & Second K-S Test \\
\hline 2 & 50 & .9958 & 1.0000 \\
\hline 2 & 100 & .9921 & 1.0000 \\
\hline 2 & 150 & .8820 & 1.0000 \\
\hline 2 & 200 & .6107 & 1.0000 \\
\hline 2 & 300 & .7762 & 1.0000 \\
\hline 2 & 500 & .8110 & 1.0000 \\
\hline 2 & 700 & .7562 & 1.0000 \\
\hline 2 & 900 & .5342 & 1.0000 \\
\hline 3 & 50 & .6779 & 1.0000 \\
\hline 3 & 100 & .8000 & 1.0000 \\
\hline 3 & 150 & .8738 & 1.0000 \\
\hline 3 & 200 & .7318 & 1.0000 \\
\hline 3 & 300 & .6107 & 1.0000 \\
\hline 3 & 500 & 6019 & 1.0000 \\
\hline 3 & 700 & .5765 & 1.0000 \\
\hline 3 & 900 & .1400 & 1.0000 \\
\hline 5 & 50 & .9541 & 1.0000 \\
\hline 5 & 100 & .4431 & 1.0000 \\
\hline 5 & 150 & .0718 & 1.0000 \\
\hline 5 & 200 & .5272 & 1.0000 \\
\hline 5 & 300 & .1386 & 1.0000 \\
\hline 5 & 500 & .2184 & 1.0000 \\
\hline 5 & 700 & .1753 & 1.0000 \\
\hline 5 & 900 & .0305 & 1.0000 \\
\hline 10 & 50 & .0021 & 1.0000 \\
\hline 10 & 100 & .0082 & 1.0000 \\
\hline 10 & 150 & $1.8654 e-009$ & 1.0000 \\
\hline 10 & 200 & $1.4725 e-005$ & 1.0000 \\
\hline 10 & 300 & $9.6681 e-009$ & 1.0000 \\
\hline 10 & 500 & $5.6889 e-010$ & 1.0000 \\
\hline 10 & 700 & $2.3889 e-020$ & 1.0000 \\
\hline 10 & 900 & $7.6473 e-024$ & 1.0000 \\
\hline 50 & 50 & $2.1647 e-023$ & 1.0000 \\
\hline 50 & 100 & $6.6643 e-019$ & 1.0000 \\
\hline 50 & 150 & $3.3610 e-037$ & 1.0000 \\
\hline 50 & 200 & $2.1543 e-027$ & 1.0000 \\
\hline 50 & 300 & $7.2496 e-067$ & 1.0000 \\
\hline 50 & 500 & $1.2755 e-069$ & 1.0000 \\
\hline 50 & 700 & $1.1159 e-107$ & 1.0000 \\
\hline 50 & 900 & $6.2242 e-131$ & 1.0000 \\
\hline 100 & 50 & $2.0685 e-017$ & 1.0000 \\
\hline 100 & 100 & $1.5506 e-045$ & 1.0000 \\
\hline 100 & 150 & $6.3211 e-059$ & 1.0000 \\
\hline 100 & 200 & $2.3167 e-055$ & 1.0000 \\
\hline
\end{tabular}


(a) Continued.

\begin{tabular}{lccc}
\hline \multicolumn{2}{c}{ Star Network } & \multicolumn{2}{c}{$P$-value } \\
No. of firms & No. of revenue scenarios & First K-S Test & Second K-S Test \\
\hline 100 & 300 & $6.1185 e-074$ & 1.0000 \\
100 & 500 & $3.0720 e-090$ & 1.0000 \\
100 & 700 & $9.3211 e-128$ & 1.0000 \\
100 & 900 & $2.6643 e-183$ & 1.0000 \\
200 & 50 & $4.0089 e-015$ & 1.0000 \\
200 & 100 & $1.5506 e-045$ & 1.0000 \\
200 & 150 & $1.6517 e-028$ & 1.0000 \\
200 & 200 & $1.4175 e-089$ & 1.0000 \\
200 & 300 & $8.0437 e-119$ & 1.0000 \\
200 & 500 & $1.0424 e-097$ & 1.0000 \\
200 & 700 & $1.6714 e-125$ & 1.0000 \\
200 & 900 & $6.3297 e-203$ & 1.0000 \\
\hline
\end{tabular}

(b)

\begin{tabular}{|c|c|c|c|}
\hline \multicolumn{2}{|c|}{ Series Network } & \multicolumn{2}{|c|}{$P$-value } \\
\hline No. of firms & No. of revenue scenarios & First K-S Test & Second K-S Test \\
\hline 2 & 50 & .8409 & 1.0000 \\
\hline 2 & 100 & .9610 & 1.0000 \\
\hline 2 & 150 & .2737 & 1.0000 \\
\hline 2 & 200 & .9596 & 1.0000 \\
\hline 2 & 300 & .9957 & 1.0000 \\
\hline 2 & 500 & .9572 & 1.0000 \\
\hline 2 & 700 & .9342 & 1.0000 \\
\hline 2 & 900 & .8418 & 1.0000 \\
\hline 3 & 50 & .9958 & 1.0000 \\
\hline 3 & 100 & .0994 & 1.0000 \\
\hline 3 & 150 & .7055 & 1.0000 \\
\hline 3 & 200 & .5272 & 1.0000 \\
\hline 3 & 300 & .0934 & 1.0000 \\
\hline 3 & 500 & .6019 & 1.0000 \\
\hline 3 & 700 & .8384 & 1.0000 \\
\hline 3 & 900 & .7702 & 1.0000 \\
\hline 5 & 50 & .8409 & 1.0000 \\
\hline 5 & 100 & .8938 & 1.0000 \\
\hline 5 & 150 & .8000 & 1.0000 \\
\hline 5 & 200 & .3767 & 1.0000 \\
\hline 5 & 300 & .6397 & 1.0000 \\
\hline 5 & 500 & .8567 & 1.0000 \\
\hline 5 & 700 & .8748 & 1.0000 \\
\hline 5 & 900 & .0173 & 1.0000 \\
\hline 10 & 50 & .6779 & 1.0000 \\
\hline 10 & 100 & .7942 & 1.0000 \\
\hline 10 & 150 & .5272 & 1.0000 \\
\hline 10 & 200 & .6397 & 1.0000 \\
\hline 10 & 300 & .3762 & 1.0000 \\
\hline 10 & 500 & .2208 & 1.0000 \\
\hline
\end{tabular}


(b) Continued.

\begin{tabular}{|c|c|c|c|}
\hline \multicolumn{2}{|c|}{ Star Network } & \multicolumn{2}{|c|}{$P$-value } \\
\hline No. of firms & No. of revenue scenarios & First K-S Test & Second K-S Test \\
\hline 10 & 700 & .1672 & 1.0000 \\
\hline 10 & 900 & .0349 & 1.0000 \\
\hline 50 & 50 & $2.1647 e-023$ & 1.0000 \\
\hline 50 & 100 & $9.1220 e-009$ & 1.0000 \\
\hline 50 & 150 & $2.6562 e-007$ & 1.0000 \\
\hline 50 & 200 & $7.6668 e-010$ & 1.0000 \\
\hline 50 & 300 & $4.7542 e-005$ & 1.0000 \\
\hline 50 & 500 & .0555 & 1.0000 \\
\hline 50 & 700 & $8.9159 e-004$ & 1.0000 \\
\hline 50 & 900 & .0025 & 1.0000 \\
\hline 100 & 50 & $2.9719 e-009$ & 1.0000 \\
\hline 100 & 100 & $1.5506 e-045$ & 1.0000 \\
\hline 100 & 150 & $5.8193 e-007$ & 1.0000 \\
\hline 100 & 200 & $5.4170 e-009$ & 1.0000 \\
\hline 100 & 300 & $9.6737 e-009$ & 1.0000 \\
\hline 100 & 500 & $1.3138 e-009$ & 1.0000 \\
\hline 100 & 700 & $8.7499 e-010$ & 1.0000 \\
\hline 100 & 900 & $9.0278 e-007$ & 1.0000 \\
\hline 200 & 50 & $1.0799 e-008$ & 1.0000 \\
\hline 200 & 100 & $3.6964 e-012$ & 1.0000 \\
\hline 200 & 150 & $1.3332 e-024$ & 1.0000 \\
\hline 200 & 200 & $1.4175 e-089$ & 1.0000 \\
\hline 200 & 300 & $1.4008 e-034$ & 1.0000 \\
\hline 200 & 500 & $1.2495 e-021$ & 1.0000 \\
\hline 200 & 700 & $3.3716 e-014$ & 1.0000 \\
\hline 200 & 900 & $3.6571 e-022$ & 1.0000 \\
\hline
\end{tabular}

(c)

\begin{tabular}{lccc}
\hline \multicolumn{2}{c}{$\begin{array}{c}\text { Star Network } \\
\text { No. of revenue scenarios }\end{array}$} & First K-S Test & $P$-value \\
\hline 12 & 50 & $2.9719 e-009$ & Second K-S Test \\
12 & 100 & $9.1220 e-009$ & 1.0000 \\
12 & 150 & $1.9937 e-013$ & 1.0000 \\
12 & 200 & $2.4000 e-011$ & 1.0000 \\
12 & 300 & $8.6264 e-023$ & 1.0000 \\
12 & 500 & $1.1180 e-034$ & 1.0000 \\
12 & 700 & $1.3228 e-046$ & 1.0000 \\
12 & 900 & $1.2521 e-042$ & 1.0000 \\
\hline
\end{tabular}

\subsubsection{Triangular or "Full" Network}

In this type of network, firm $m$ makes loans to firms $1,2, \ldots, m-1$. Firm $m-1$ loans to firms $1,2, \ldots, m-2$, and so on. The network equations are then (see also Figure 2 ) 


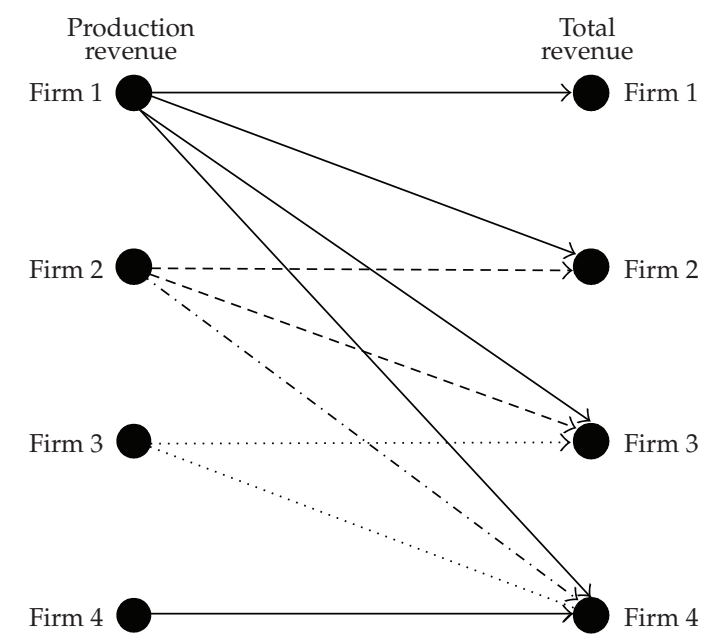

Figure 2: A Triangular or "Full" network.

$$
\begin{gathered}
\alpha_{1}(\omega)=v_{1}(\omega), \\
\alpha_{2}(\omega)=\lambda_{12} * v_{1}(\omega)+v_{2}(\omega), \\
\alpha_{3}(\omega)=\lambda_{13} * v_{1}(\omega)+\lambda_{23} * v_{2}(\omega)+v_{3}(\omega) \\
\vdots \\
\alpha_{m}(\omega)=\lambda_{1 m} * v_{1}(\omega)+\lambda_{2 m} * v_{2}(\omega)+\cdots+\lambda_{m-1, m} * v_{m-1}(\omega)+v_{m}(\omega)
\end{gathered}
$$

We analyze the specific case $\lambda_{i, j}=0.5$ for $i<j$. Also, all the firms have the same size, that is, $E\left[v_{i}\right]=1$.

\subsubsection{Series Network}

In a series network, each firm $m-1$ borrows from the "next" firm $m$. It is illustrated in Figure 3:

$$
\begin{gathered}
\alpha_{1}(\omega)=v_{1}(\omega), \\
\alpha_{2}(\omega)=0.5 \alpha_{1}(\omega)+v_{2}(\omega), \\
\alpha_{3}(\omega)=0.5 \alpha_{2}(\omega)+v_{3}(\omega), \\
\alpha_{4}(\omega)=0.5 \alpha_{3}(\omega)+v_{4}(\omega), \\
\vdots \\
\alpha_{m}(\omega)=0.5 \alpha_{m-1, m}(\omega)+v_{m}(\omega),
\end{gathered}
$$

where $\lambda_{m-1, m}=0.5$. 


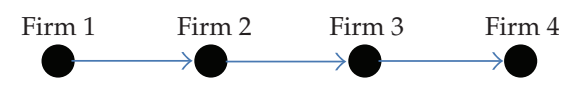

Figure 3: A Series network.

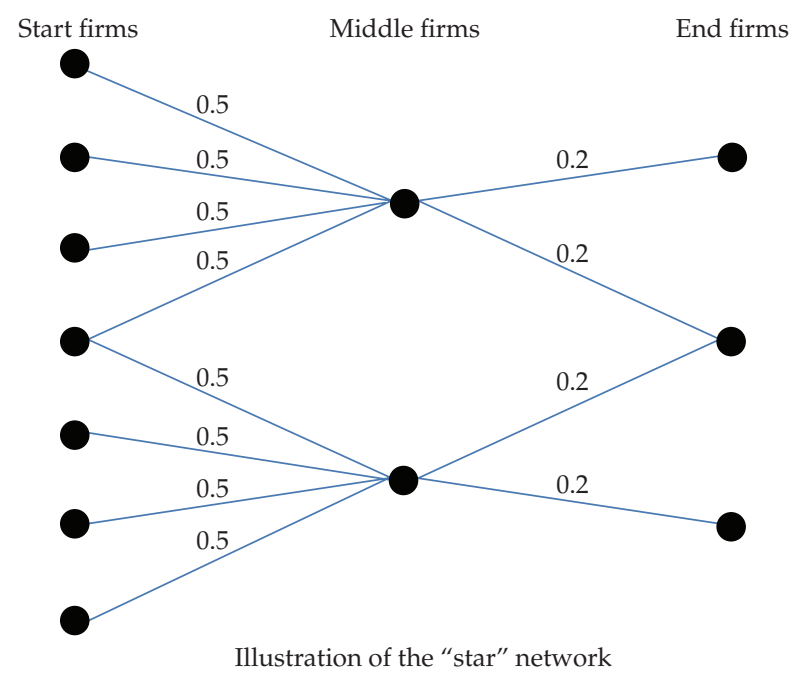

Figure 4: The "Star" network.

\subsubsection{Star Network}

A star network is an idealization of an economy with a small group of large "middle firms" who both lend to a set of "start firms" and borrow from a set of "end firms." Start and end firms all have the same revenue size, that is, $E\left[v_{i}\right]=1$. All large firms also have the same revenue size, $E\left[v_{i}\right]=5$.

We choose a particular network with 7 start firms, 2 middle firms, and 3 end firms. A typical example is the US car industry, with Ford and GM as "middle firms", dealers as end firms, and suppliers as start firms. Figure 4 illustrates this type of network. Since only the production revenue of a (say) start firm times lambda matters in determining the impact of a start firm on a middle firm, it is needless to vary lambda in this network to account for different relative impact of start firms on middle firms. A different relative revenue suffices.

\subsection{Distributional Assumptions}

Our main assumption is that the dependence between production revenues is described by the Gaussian copula. Since copulae are invariant under monotone transformations of variables, the copula of the logarithm of revenue is also Gaussian under this hypothesis. We chose the following variance-covariance matrix for $v$ :

$$
\operatorname{Var}\left[v v^{t}\right]=\left[\begin{array}{llll}
1 & \rho & \cdots & \rho \\
\rho & 1 & & \cdot \\
\cdot & & & \rho \\
\rho & \cdot & \rho & 1
\end{array}\right]
$$


with $\rho=0.5$. This choice was fairly arbitrary, but we found out in other tests (not reported here) that other values of $\rho$ give us similar results. We test different types of marginal distributions $F$ for production revenue $\widehat{v}_{i}$ :

(i) exponential with various rates, namely, 1, 5, and 20

(ii) uniform with various support,

(iii) gamma with various shapes and scales,

(iv) quadratic $F(x)=x^{2}$ where $x \in[0,1]$,

(v) cubic $F(x)=x^{3}$ where $x \in[0,1]$.

Exponential, gamma, and uniform random variables are standard choices for modelling positive random variables (see, e.g., Lando [9]).

\section{Statistical Methodology}

As stated earlier, the main goal in this paper was to compare t-copula with a Gaussian copula as a way to model counterparty risk. Since the Gaussian copula is a special case of a t-copula, namely a t-copula with an infinite number of degrees of freedom, we try to fit a t-copula to our simulated data. The calibrated number of degrees of freedom will be a good indicator whether a nonGaussian $t$-copula is a better choice than the Gaussian copula. We then expose our methodology for hypothesis testing.

\subsection{Parameter Estimation}

Assuming that the dependence of the firms' counterparty risk premia is the Student's tcopula, we conduct our estimation analyses based on the IFM method discussed in Chapter 5.3 of the book Copula Methods in Finance by Cherubini et al. [1]. Cherubini et al's method is composed of two following steps:

(1) infer the parameter(s) $\theta_{1}$ of the marginals,

(2) infer the parameter(s) $\theta_{2}$ of the Student's t-copula.

Our analyses focus on the second step: estimating the parameter(s) of the copula $\theta_{2}$. We bypass the first step because we take the empirical distribution of the marginals as given. The Student's t-copula has two parameters, namely, the correlation matrix $R$ and the degrees of freedom $v$. For simplicity, we use the method of moments to first infer the correlation matrix, and use maximum likelihood to estimate the degrees of freedom $v$ :

$$
\widehat{v}=\arg \max _{v} \sum_{\omega=1}^{\Omega} \ln c_{R, v}\left(F_{1}\left(\alpha_{\omega, 1}\right), F_{2}\left(\alpha_{\omega, 2}\right), \ldots, F_{n}\left(\alpha_{\omega, m}\right)\right)
$$

where

$$
\begin{gathered}
c_{R, v}\left(u_{1}, u_{2}, \ldots, u_{m}\right)=|R|^{-1 / 2} \frac{\Gamma((v+2) / 2)}{\Gamma(v / 2)}\left(\frac{\Gamma(v / 2)}{\Gamma((v+1) / 2)}\right)^{2} \frac{\left(1+(1 / v) \varsigma^{t} R^{-1} s\right)^{-(v+2) / 2}}{\prod_{j=1}^{m}\left(1+\varsigma_{j}^{2} / v\right)^{-(v+1) / 2}} \\
\varsigma_{j}=t_{v}^{-1}\left(u_{j}\right) .
\end{gathered}
$$


Let $m$ be the total number of firms in the network and $\Omega$ the total number of revenue scenarios. The following algorithm constructs the network economy and estimates the parameter vector $v$.

(1) Generate a table of total revenues $\alpha$ based on the relationship specified in each network discussed in the previous section:

$$
\begin{gathered}
\alpha_{11}, \alpha_{21}, \ldots, \alpha_{\Omega 1}, \\
\alpha_{12}, \alpha_{22}, \ldots, \alpha_{\Omega 2}, \\
\vdots \\
\alpha_{1 m}, \alpha_{2 m}, \ldots, \alpha_{\Omega m} .
\end{gathered}
$$

(2) Calculate, for each scenario $\omega$,

$$
\begin{aligned}
& \widehat{U}_{\omega 1}=\widehat{F}_{1}\left(\alpha_{\omega, 1}\right)=\frac{1}{\Omega} \sum_{i, k=1}^{\Omega} 1\left\{\alpha_{i, 1}<\alpha_{k, 1}\right\}, \\
& \widehat{U}_{\omega 2}=\widehat{F}_{2}\left(\alpha_{\omega, 2}\right)=\frac{1}{\Omega} \sum_{i, k=1}^{\Omega} 1\left\{\alpha_{i, 2}<\alpha_{k, 2}\right\}, \\
& \widehat{U}_{\omega m}=\widehat{F}_{2}\left(\alpha_{\omega, m}\right)=\frac{1}{\Omega} \sum_{i, k=1}^{\Omega} 1\left\{\alpha_{i, m}<\alpha_{k, m}\right\} .
\end{aligned}
$$

(3) Transform the variable $\widehat{U}$ into the variable $\zeta$ for each scenario $\omega$ :

$$
\begin{gathered}
\zeta_{\omega 1}=t_{v}^{-1}\left(\widehat{U}_{\omega 1}\right), \\
\zeta_{\omega 2}=t_{v}^{-1}\left(\widehat{U}_{\omega 2}\right), \\
\vdots \\
\zeta_{\omega m}=t_{v}^{-1}\left(\widehat{U}_{\omega m}\right) .
\end{gathered}
$$

(4) Calculate the covariance matrix of $\zeta$ :

$$
R_{\mathrm{emp}}=\operatorname{Cov}\left(\zeta_{\omega 1}, \zeta_{\omega 2}, \ldots, \zeta_{\omega m}\right)
$$


(5) Maximize over the degrees of freedom $v$ the log likelihood of the Student's t-copula density:

$$
\begin{aligned}
\max _{v} & \sum_{\omega=1}^{\Omega} \ln c\left(F_{1}\left(\alpha_{\omega, 1}\right), F_{2}\left(\alpha_{\omega, 2}\right), \ldots, F_{2}\left(\alpha_{\omega, n}\right)\right) \\
& =\max \ln \left(\left|R_{\mathrm{emp}}\right|^{-1 / 2} \frac{\Gamma((v+2) / 2)}{\Gamma(v / 2)}\left(\frac{\Gamma(v / 2)}{\Gamma((v+1) / 2)}\right)^{2} \frac{\left(1+(1 / v) \varsigma_{i}^{t} R_{\mathrm{emp}}^{-1} \varsigma_{i}\right)^{-(v+2) / 2}}{\prod_{j=1}^{n}\left(1+\varsigma_{i, j}^{2} / v\right)^{-(v+1) / 2}}\right),
\end{aligned}
$$

where

$$
\begin{gathered}
\varsigma_{\omega, 1}=t_{v}^{-1}\left(\widehat{U}_{\omega 1}\right), \\
\varsigma_{\omega, 2}=t_{v}^{-1}\left(\widehat{U}_{\omega 2}\right), \\
\vdots \\
\varsigma_{\omega, n}=t_{v}^{-1}\left(\widehat{U}_{\omega n}\right) .
\end{gathered}
$$

\subsection{Hypothesis Testing}

In the section, we describe our methodology to determine whether or not we made the right assumption, that the Student's copula fits our counterparty risk premium (simulated) data better than the Gaussian copula. The goodness-of-fit test we use to compare the empirical distribution with the hypothesized cumulative distribution is called the KolmogorovSmirnov test. We perform two K-S tests: (1) empirical versus normal and (2) empirical versus Student's $t$.

\subsubsection{First K-S Test: Empirical versus Normal}

The first K-S test rests on the fact that if the copula of the counterparty risk premium $C_{\alpha_{1}, \alpha_{2}, \ldots, \alpha_{m}}$ is normal then the distribution of the variable $z_{\text {emp }}^{2}$ (defined below) should be chi-square $x^{2}$ with degrees of freedom equal to the number of the firms in the network $m$. For a given network, we conduct the first K-S test as follows.

The hypotheses are

$$
H_{0} \text { : the copula } C_{\alpha_{1}, \alpha_{2}, \ldots, \alpha_{m}} \text { is Gaussian, }
$$

$$
H_{1} \text { : the copula } C_{\alpha_{1}, \alpha_{2}, \ldots, \alpha_{m}} \text { is not Gaussian. }
$$

We follow the methodology discussed in Malevergne and Sornette [10]. 
(1) Find the empirical distribution $\widehat{F}_{\alpha_{m}}$ of $\alpha_{m}$ and calculate $\widehat{U}_{m}$ as in the parameter estimation step:

$$
\begin{gathered}
\widehat{U}_{1}=\left(\widehat{F}_{\alpha_{1}}\left(\alpha_{1}\right)\right), \\
\vdots \\
\widehat{U}_{n}=\left(\widehat{F}_{\alpha_{n}}\left(\alpha_{m}\right)\right),
\end{gathered}
$$

where

$$
\begin{gathered}
\widehat{F}_{1}\left(\alpha_{\omega, 1}\right)=\frac{1}{\Omega} \sum_{i, k=1}^{\Omega} 1\left\{\alpha_{i, 1}<\alpha_{k, 1}\right\} \\
\vdots \\
\widehat{F}_{n}\left(\alpha_{\omega, m}\right)=\frac{1}{\Omega} \sum_{i, k=1}^{\Omega} 1\left\{\alpha_{i, m}<\alpha_{k, m}\right\} .
\end{gathered}
$$

(2) Calculate the Gaussian variables $y_{\omega 1}, y_{\omega 2}, \ldots, y_{\omega m}$ via the following transformation:

$$
\begin{gathered}
y_{\omega 1}=\Phi^{-1}\left(\widehat{U}_{\omega 1}\right)=\Phi^{-1}\left(\widehat{F}_{\alpha_{1}}\left(\alpha_{\omega 1}\right)\right), \\
\vdots \\
y_{\omega m}=\Phi^{-1}\left(\widehat{U}_{\omega n}\right)=\Phi^{-1}\left(\widehat{F}_{\alpha_{n}}\left(\alpha_{\omega m}\right)\right) .
\end{gathered}
$$

(3) Determine the covariance matrix $R_{\mathrm{emp}}$ of the Gaussian variables $y_{1}, y_{2}, \ldots, y_{m}$

(4) Calculate the variable $z_{\text {emp. }}^{2}$

$$
z_{\text {emp. }}^{2}(\omega)=y^{t} R_{\text {emp }}^{-1} y
$$

(5) Find the empirical distribution of $z_{\text {emp. }}^{2}$ namely, $\widehat{F}_{z_{\text {emp }}^{2}}$

(6) Calculate the Kolmogorov distance:

$$
D=\max _{z_{\text {emp }}^{2}}\left|\widehat{F}_{z_{\text {emp }}^{2}}\left(z_{\text {emp }}^{2}\right)-F_{x^{2}}\left(z_{\text {emp }}^{2}\right)\right| .
$$

(7) Verify the result by using the kstest2 (Kolmogorov test to compare the distribution of two samples) command in Matlab. 


\subsubsection{Second K-S Test: Empirical versus $t$}

As previously mentioned, the second K-S test will allow us to compare the empirical distribution of the copula to the theoretical Student's $t$ distribution. We will then compare the results of the second K-S test to those of the first K-S test. We proceed with the second K-S test as follows:

$$
\begin{gathered}
H_{0} \text { : the copula } C_{\alpha_{1}, \alpha_{2}, \ldots, \alpha_{m}} \text { is Student's copula, } \\
H_{1} \text { : the copula } C_{\alpha_{1}, \alpha_{2}, \ldots, \alpha_{m}} \text { is not Student's copula. }
\end{gathered}
$$

Unlike in the Gaussian copula case, there is no analytical expression for the density of the theoretical Kolmogorov-Smirnov statistic $z_{\text {th. }}^{2}$ in the $\mathrm{t}$-copula case. Thus we generate by simulation (over a very large number of scenarios $\bar{\Omega}$ ) the distribution $\widehat{F}_{z_{\mathrm{th}}^{2}}$. The overall algorithm is as follows.

(1) Generate the "theoretical" data $\left(x_{\omega 1}, x_{\omega 2}, \ldots, x_{\omega m}\right)$ by Conditional Monte Carlo (CMC) simulation following the method adapted from that of Aas et al. [11] and find the empirical distribution $\widehat{F}_{x_{m}}$ of the theoretical data. Please refer to the appendix for the Conditional Monte Carlo (CMC) simulation algorithm.

(2) Calculate $\widehat{U}_{m}$ :

$$
\begin{gathered}
\widehat{U}_{1}^{\text {th }}=\left(\widehat{F}_{x_{1}}\left(x_{1}\right)\right), \\
\vdots \\
\widehat{U}_{m}^{\text {th }}=\left(\widehat{F}_{x_{m}}\left(x_{m}\right)\right) .
\end{gathered}
$$

(3) Calculate the variables $y_{\omega 1}^{\text {th }}, y_{\omega 2}^{\text {th }}, \ldots, y_{\omega n}^{\text {th }}$

$$
\begin{gathered}
y^{\text {th }}(\omega, 1)=\Phi^{-1}\left(\widehat{U}_{1}^{\text {th }}(\omega)\right), \\
\vdots \\
y^{\text {th }}(\omega, m)=\Phi^{-1}\left(\widehat{U}_{m}^{\text {th }}(\omega)\right) .
\end{gathered}
$$

(4) Determine the covariance matrix $R_{\text {th }}$ of the variables $y_{1}^{\text {th }}, y_{2}^{\text {th }}, \ldots, y_{m}^{\text {th }}$.

(5) Calculate the variable $z_{\mathrm{th}}^{2}$ :

$$
z_{\text {th. }}^{2}(\omega)=\left(y^{\text {th }}\right)^{t} R_{\text {th }}^{-1} y^{\text {th }}
$$

(6) Find the theoretical distribution of $z_{\mathrm{th} .}^{2}$, namely, $\widehat{F}_{z_{\mathrm{th}}^{2}}$ : 


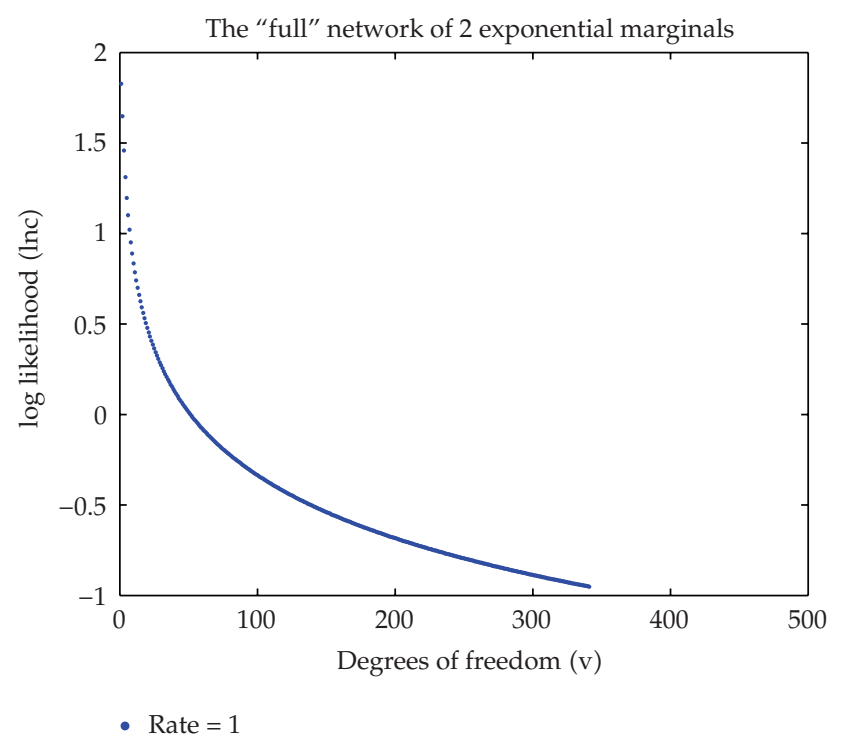

Figure 5: Inference on the triangular network with 2 firms. Marginal distribution is exponential with $E\left[v_{i}\right]=1$.

(7) Calculate the Kolmogorov distance

$$
D=\max _{z_{\text {emp }}^{2}}\left|\widehat{F}_{z_{\text {emp }}^{2}}\left(z_{\text {emp }}^{2}\right)-F_{z_{\text {th }}^{2}}\left(z_{\text {emp }}^{2}\right)\right|
$$

(8) Verify the result by using the kstest2 command in Matlab.

\section{Results}

\subsection{Parameter Estimation}

The principal result that we obtain is that, for counterparty risk premia, any Student's tcopula results in a better fit than the Gaussian copula.

We show the log-likelihood defined on the right-hand-side of (3.1) as a function of the number of degrees of freedom of the Student's $t$ distribution. In our simulation, we use various numbers of scenarios: 50, 100, 150, 200, 300, 500, 700 to 900 scenarios. For the triangular and series networks, the number of firms varies from 2, 3, 5, 10, 20, 50, 100 to 200. In all these cases, the log-likelihood function is uniformly decreasing. In other words, the degrees of freedom that maximize the log-likelihood are finite numbers in all the cases.

We show hereafter a subset of our simulation results. In Figures 5 to 16 are shown the results for the triangular, or "full" network. In Figure 17, are shown the results for the series network, while in Figure 18 we show results for the star network. The benchmark marginal distribution is exponential. For the triangular network, we tried also all the marginal distributions specified earlier in order to verify that the marginal distributions of the firms' production revenues have no effect on the dependence structure of the their counterparty risk premia. 


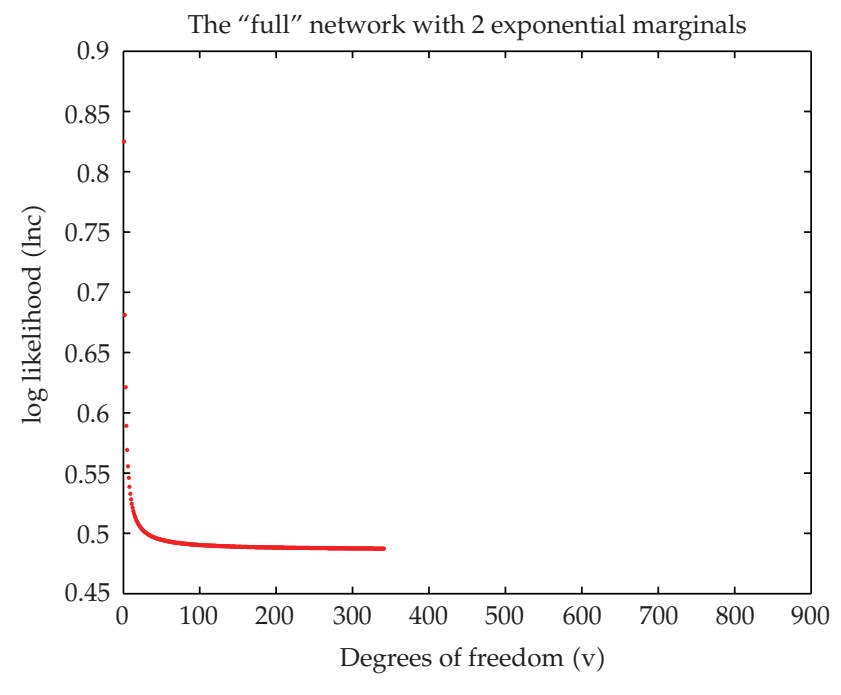

- Rate $=5$

Figure 6: Inference on the triangular network with 2 firms. Marginal distribution is exponential with $E\left[v_{i}\right]=5$.

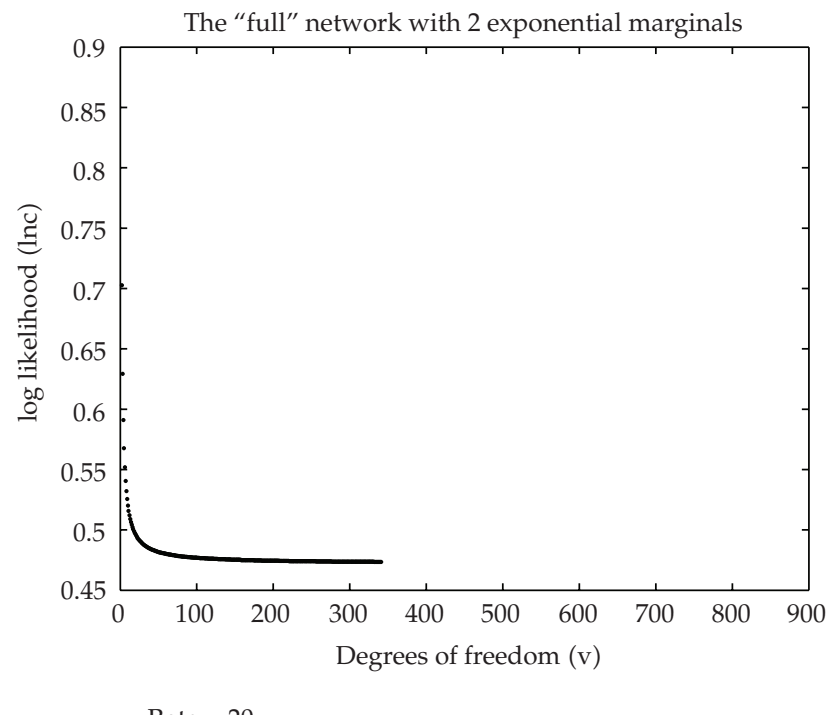

Figure 7: Inference on the triangular network with 2 firms. Marginal distribution is exponential with $E\left[v_{i}\right]=20$.

It is worth mentioning again that the log-likelihood function is uniformly decreasing across all samples. As mentioned above, the Gaussian distribution is a Student's $t$-distribution with an infinite number of degrees of freedom. Thus, from our parameter estimation step, we conclude that any Student's $t$-distribution results in a better fit than the Gaussian distribution. This fact will be confirmed when we perform hypothesis testing for goodness-of-fit check. 


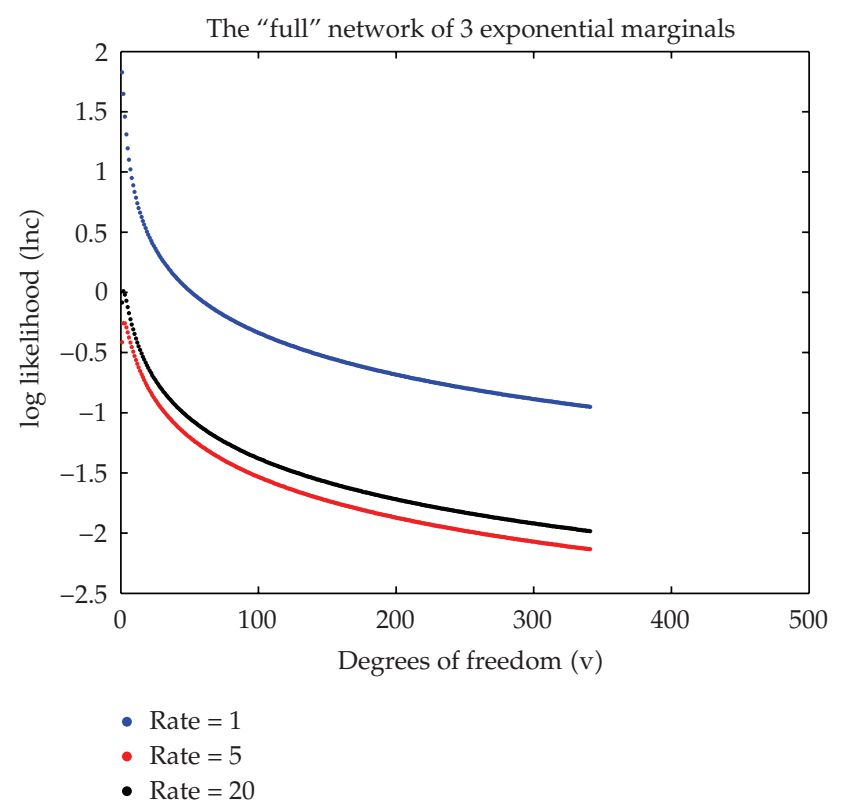

Figure 8: Inference on the triangular network with 3 firms. Marginal distribution is exponential.

\subsection{1. "Full" or Triangular Networks}

See Figures 5-16.

\subsubsection{Other Networks}

See Figures 17 and 18.

\subsection{Hypothesis Testing}

\subsubsection{First K-S Test: Empirical versus Normal}

We analyze the Kolmogorov statistic $D$ which represents the maximum distance between the empirical distribution $\widehat{F}_{z_{\text {emp }}^{2}}$ and the $x^{2}$-distribution.

As in the parameter estimation step, we varied our revenue scenarios from 50, 100, 150, 200, 300, 500, 700 to 900 scenarios and the number of firms from 2, 3, 5, 10, 20, 50, 100 to 200 in each of our networks. As the number of firms in the triangular network increases, the Kolmogorov statistic $D$ increases, thus allowing us to reject the null hypothesis that the copula of the counterparty risk premium is Gaussian if the number of firms is sufficiently large. We also use Matlab's kstest2 function to verify the reliability of our Kolmogorov statistic $D$. Note that our Kolmogorov statistic $D$ takes into account only the maximum difference value while the Matlab's kstest2 function takes into account the difference values at all data points between the empirical distribution and the theoretical distribution $\left(x^{2}\right.$, in this case). The Matlab's kstest2 results are consistent with our conclusion. Specifically, when the number of firms in the network exceeds 10, we reject the null hypothesis in favor of the 


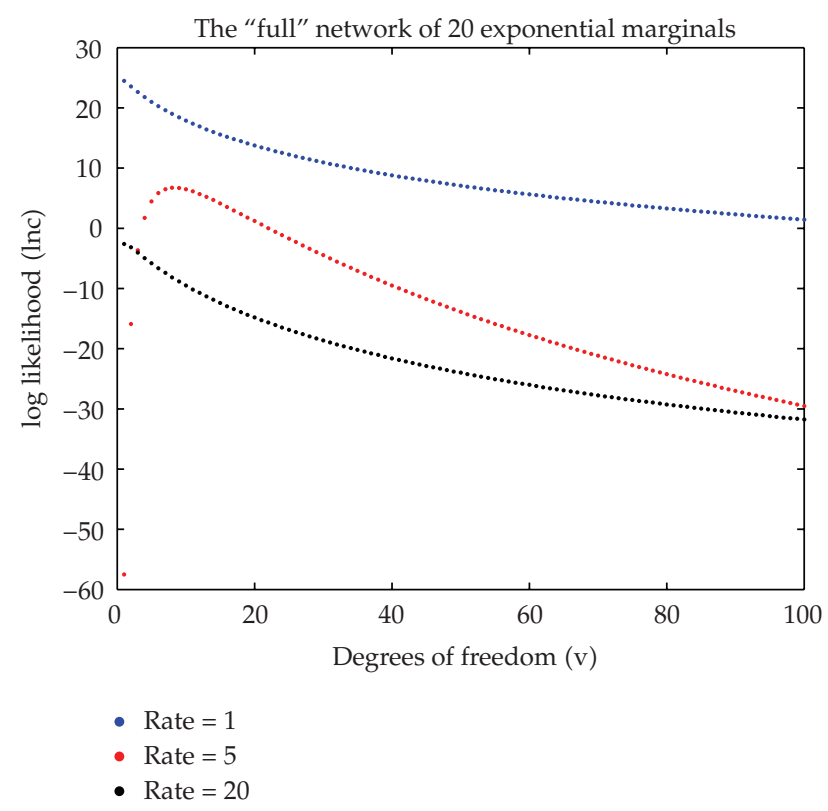

Figure 9: Inference on the triangular network with 4 firms. Marginal distribution is exponential.

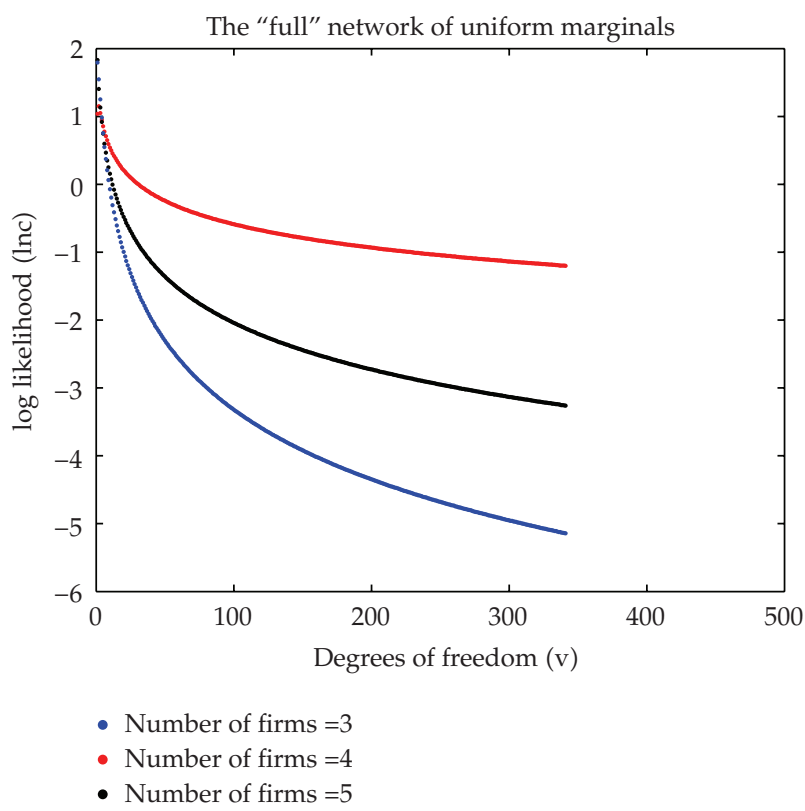

Figure 10: Inference on the triangular network with 2, 3, and 4 firms. Marginal distribution is standard uniform.

alternative hypothesis at a 5\% significance level. In plain words, when the number of firms exceeds 10 in the network, the copula that captures the dependence of the counterparty risk premium is not Gaussian.

Similar results hold for the series and "star" networks. In the series network, when the number of firms in the network exceeds 30, we reject the null hypothesis that the copula is 


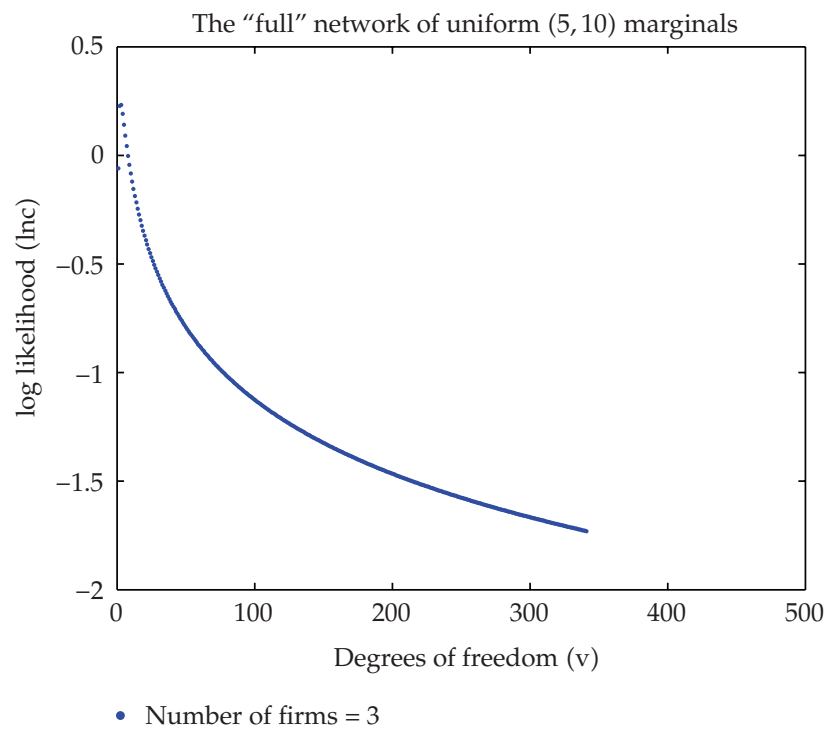

Figure 11: Inference on the triangular network with 2 firms. Marginal distribution is uniform with support $(5,10)$.

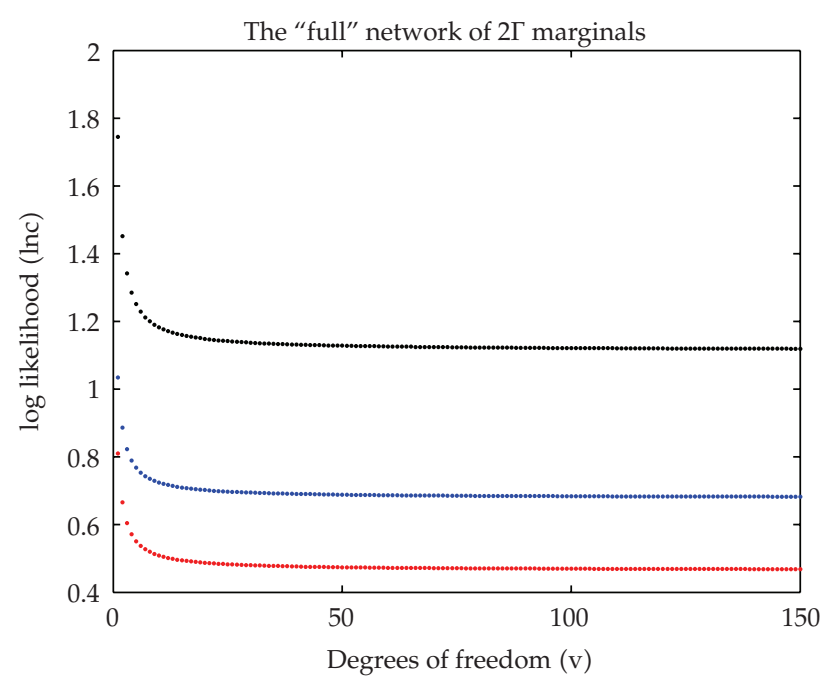

- Shape $=1$, scale $=2$

- Shape $=2$, scale $=2$

- Shape $=9$, scale $=0.5$

Figure 12: Inference on the triangular network with 2 firms. Distribution is gamma.

Gaussian. We also reject the null hypothesis in the "star" network of 12 firms. In sum, when the number of the firms increases, we are more likely to reject the null hypothesis. This leads us to conclude that as the number of firms increases, the copula that captures the dependence of the counterparty risk premium is more likely to be nonGaussian. 


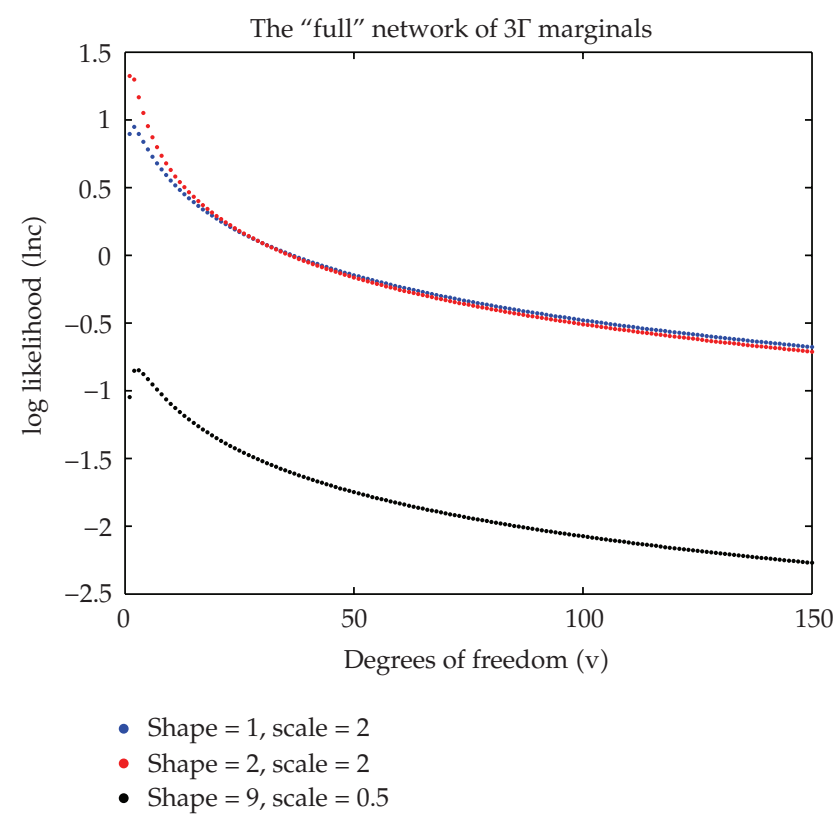

Figure 13: Inference on the triangular network with 3 firms. Marginal distribution is gamma.

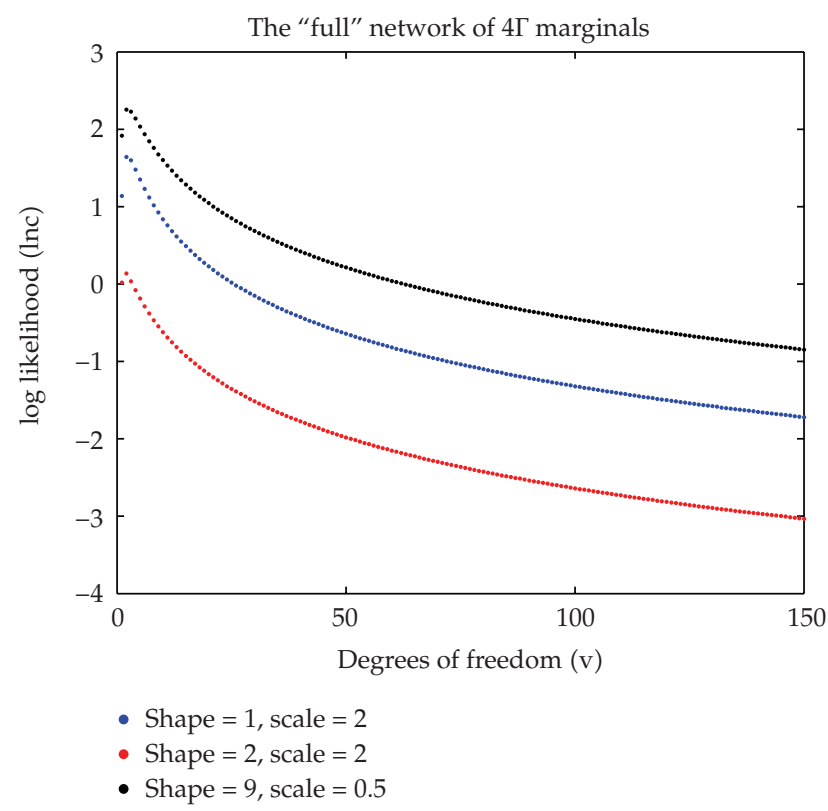

Figure 14: Inference on the triangular network with 4 firms. Marginal distribution is gamma.

\subsubsection{Second K-S Test: Empirical versus $t$}

Note that the copula is likely to be nonGaussian, is it a Student's t-copula? Which copula, Gaussian or Student's $t$, is more likely? The second K-S test will allow us to compare the empirical distribution of the copula to the theoretical Student's $t$ distribution. 


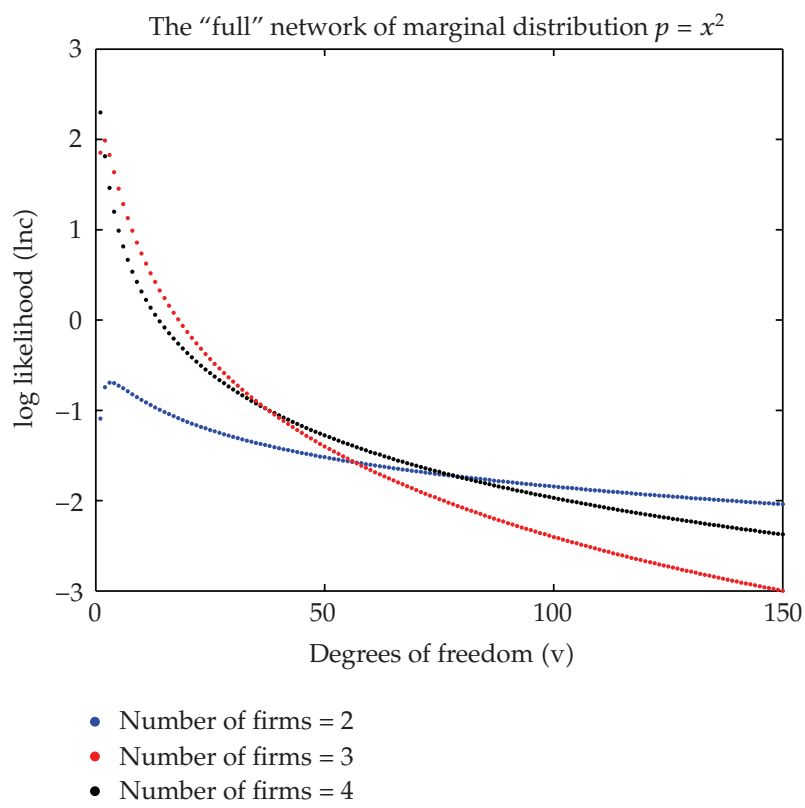

Figure 15: Inference on the triangular network. Marginal distribution is quadratic.

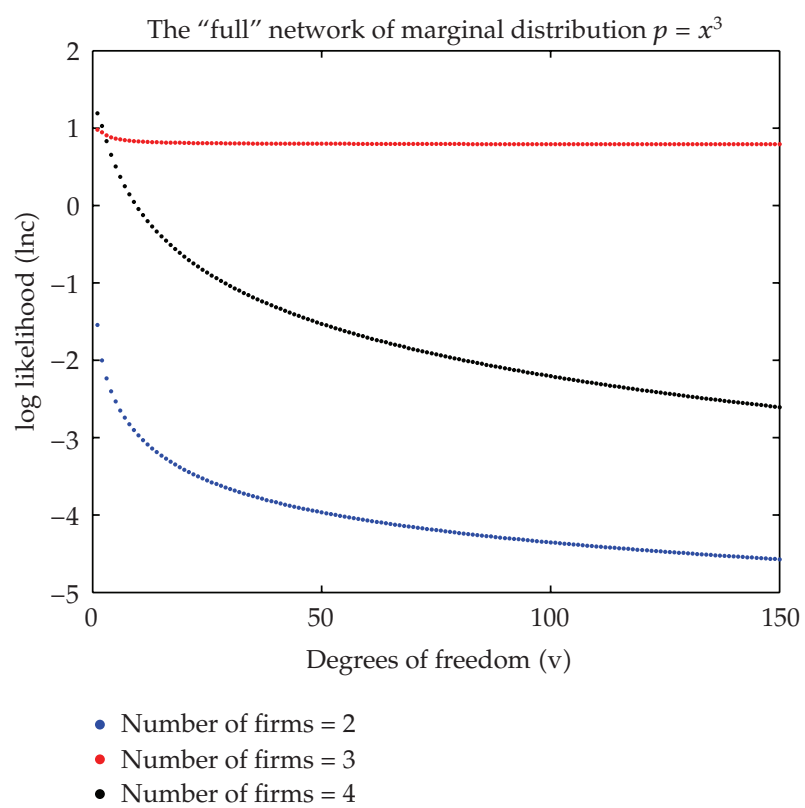

Figure 16: Inference on the triangular network with 4 firms. Marginal distribution is cubic.

As before, we did the test on the triangular or "full" network, the series network, and the "star" network. For each type of network, we varied the number of revenue scenarios from 50, 100, 150, 200, 300, 500, 700 to 900 scenarios and the number of firms from 2, 3, 5, 10, 20, 50, 100 to 200 firms. The results we obtained indicate that we cannot reject the hypothesis that the copula is Student's $t$ in all cases at the $5 \%$ significance level. In other words, we 


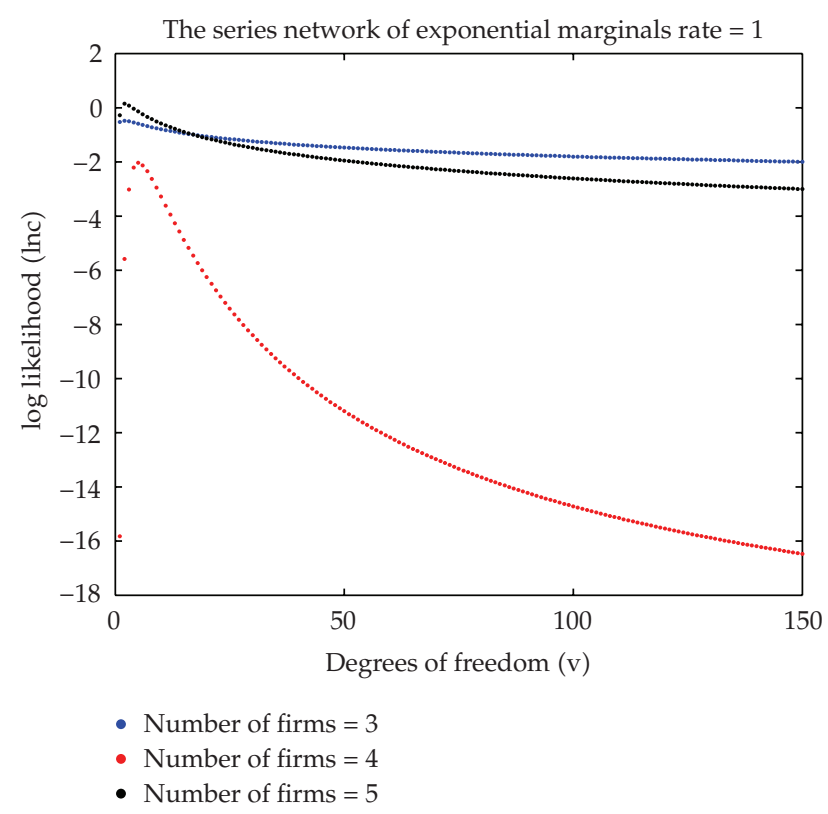

Figure 17: Inference on the series network. Marginal distribution is exponential with $E\left[v_{i}\right]=1$.

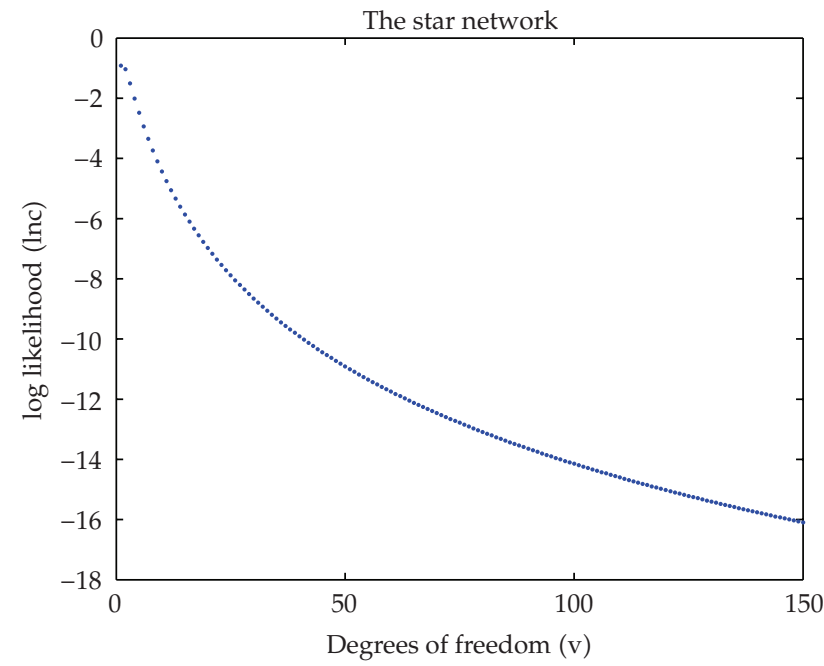

Figure 18: Inference on the star network. Marginal distribution is exponential with $E\left[v_{i}\right]=1$.

do not have sufficient evidence to reject that the copula capturing the dependence of the counterparty risk premium is Student's $t$.

\subsubsection{P-Value}

For networks of 2-5 firms, the $P$-values indicate that we cannot reject the null hypothesis in neither the first nor the second Kolmogorov-Smirnov tests. However, the $P$-values resulting 
from the first K-S test (which tests whether the empirical distribution comes from the normal distribution) are lower than the $P$-values resulting from the second K-S test (which tests whether the empirical distribution comes from the Student's $t$ distribution) in all cases. For networks of more than 10 firms, the $P$-values indicate that we can reject the null hypothesis in the first K-S test but not the second K-S test. In other words, in networks of more than 10 firms, we have sufficient evidence to conclude that the empirical distribution does not come from the normal distribution but we do not have sufficient evidence to conclude that the empirical distribution does not come from the Student's $t$ distribution.

\subsubsection{Test Power}

It is well known that the one-sided Kolmogorov-Smirnov test is superior in many aspects to the traditional goodness-of-fit test: see, for instance, Massey [12]. The latter paper provides a figure, from which the power of that test is given. For two-sided Kolmogorov-Smirnov tests, much less is known (see however Milbrodt and Strasser [13]). Given the complexity of calculating the power of that test, we doubt that many practioners engage in it, especially since more powerful tests probably do not exist.

\section{Conclusion}

In our study, we use the copula method to model the dependence between the counterparty risk premia of various firms. Specifically, we study the impact of the dependence among production revenues on the dependence among counterparty risk premia in several different network economies.

Taking as given that the dependence between the production revenues $v$ was a Gaussian copula, we generate the production revenues by simulation. Then we calculate the total revenues from the simulated production revenues. Two main tests-the parameter estimation and hypothesis testing-are carried out on each network setup. In the first test, the parameter of the copula being estimated is the number of degrees of freedom. The estimation results obtained from each network indicate that the Student's t-copula is likely to be the copula capturing the relationship between the firms' counterparty risk premia, reaffirming our assumption. The results from our second test-the Kolmogorov-Smirnov hypothesis testing-confirms our conclusion.

\section{Appendices}

Conditional Monte Carlo (CMC) simulation follows the method adapted from that of Aas et al. [11]

\section{A. Generation of the Theoretical Data}

To generate the "theoretical" sample data $\left(x_{\omega 1}, x_{\omega 2}, x_{\omega 3}, \ldots, x_{\bar{\Omega} m}\right)$ from the Student's copula, we use conditional Monte Carlo (CMC) simulation. Provided that multivariate data can be modelled using a set of pair-copulae which act on two variables at a time, we generate samples $\left(x_{\omega 1}, x_{\omega 2}, x_{\omega 3}, \ldots, x_{\bar{\Omega} m}\right)$ from the Student's copula by way of conditional Monte Carlo simulation. 
Aas et al. [11] provide a way to simplify the copula function in order to derive an efficient simulation algorithm as follows. We define

$$
\begin{aligned}
C_{12}\left(u_{1}, u_{2}\right)= & \int_{-\infty}^{t_{v_{12}}^{-1}\left(u_{1}\right)} \int_{-\infty}^{t_{v_{12}}^{-1}\left(u_{2}\right)} \frac{\Gamma\left(\left(v_{12}+2\right) / 2\right)}{\Gamma\left(v_{12} / 2\right) \sqrt{\left(\pi v_{12}\right)^{2}\left(1-\rho_{12}^{2}\right)}} \\
& \times\left\{1+\frac{x^{2}-2 \rho_{12} x y+y^{2}}{v_{12}\left(1-\rho_{12}^{2}\right)}\right\}^{-\left(v_{12}+2\right) / 2} d x d y, \\
g(x, y) & =\frac{\Gamma\left(\left(v_{12}+2\right) / 2\right)}{\Gamma\left(v_{12} / 2\right) \sqrt{\left(\pi v_{12}\right)^{2}\left(1-\rho_{12}^{2}\right)}}\left\{1+\frac{x^{2}-2 \rho_{12} x y+y^{2}}{v_{12}\left(1-\rho_{12}^{2}\right)}\right\}^{-\left(v_{12}+2\right) / 2}, \\
f_{v}(x) & =\frac{\Gamma((v+1) / 2)}{\Gamma(v / 2) \sqrt{(\pi v)}}\left(1+x^{2} / v\right)^{-(v+1) / 2}, \\
b_{1} & =t_{v_{12}}^{-1}\left(u_{1}\right) \\
b_{2} & =t_{v_{12}}^{-1}\left(u_{2}\right) .
\end{aligned}
$$

We can then calculate

$$
\begin{aligned}
& h_{12}\left(u_{1}, u_{2}\right)=F_{1 \mid 2}\left(u_{1}, u_{2}\right) \\
& =\frac{\partial}{\partial u_{2}} C_{12}\left(u_{1}, u_{2}\right) \\
& =\frac{\partial}{\partial u_{2}} \int_{-\infty}^{b_{1}} \int_{-\infty}^{b_{2}} g(x, y) d x d y \\
& =\frac{\partial b_{2}}{\partial u_{2}} \frac{\partial}{\partial b_{2}} \int_{-\infty}^{b_{1}} \int_{-\infty}^{b_{2}} g(x, y) d x d y \\
& =\frac{1}{f_{v_{12}}\left(b_{2}\right)} \int_{-\infty}^{b_{1}}\left[\frac{\partial}{\partial b_{2}} \int_{-\infty}^{b_{2}} g(x, y) d x\right] d y \\
& =\frac{1}{f_{v_{12}}\left(b_{2}\right)} \int_{-\infty}^{b_{1}} \frac{\Gamma\left(\left(v_{12}+2\right) / 2\right)}{\Gamma\left(v_{12} / 2\right) \sqrt{\left(\pi v_{12}\right)^{2}\left(1-\rho_{12}^{2}\right)}} \\
& \times\left\{1+\frac{x^{2}-2 \rho_{12} x b_{2}+b_{2}^{2}}{v_{12}\left(1-\rho_{12}^{2}\right)}\right\}^{-\left(v_{12}+2\right) / 2} d x \\
& =\frac{1}{f_{v_{12}}\left(b_{2}\right)} \int_{-\infty}^{b_{1}} \frac{\Gamma\left(\left(v_{12}+2\right) / 2\right)}{\Gamma\left(v_{12} / 2\right) \sqrt{\left(\pi v_{12}\right)^{2}\left(1-\rho_{12}^{2}\right)}} \\
& \times\left\{1+\frac{\left(x-\rho_{12} b_{2}\right)^{2}}{\left(v_{12}+b_{2}\right)^{2}\left(1-\rho_{12}^{2}\right)}\right\}^{-\left(v_{12}+2\right) / 2}\left[1+\frac{b_{2}^{2}}{v_{12}}\right]^{-\left(v_{12}+2\right) / 2} d x
\end{aligned}
$$




$$
\begin{gathered}
=\frac{1}{f_{v_{12}}\left(b_{2}\right)} \frac{\Gamma\left(\left(v_{12}+1\right) / 2\right) \sqrt{\pi\left(v_{12}+b_{2}^{2}\right)\left(1-\rho_{12}^{2}\right)}}{\Gamma\left(v_{12} / 2\right) \sqrt{\left(\pi v_{12}\right)^{2}\left(1-\rho_{12}^{2}\right)}}\left[1+\frac{b_{2}^{2}}{v_{12}}\right]^{-\left(v_{12}+2\right) / 2} \\
\quad \times\left[1+\frac{b_{2}^{2}}{v_{12}}\right]^{-1 / 2} \int_{-\infty}^{b_{1}} \frac{\Gamma\left(\left(v_{12}+2\right) / 2\right)}{\Gamma\left(\left(v_{12}+1\right) / 2\right) \sqrt{\pi\left(v_{12}+b_{2}^{2}\right)\left(1-\rho_{12}^{2}\right)}} \\
\times\left\{1+\frac{\left(x-\rho_{12} b_{2}\right)^{2}}{\left(v_{12}+b_{2}\right)^{2}\left(1-\rho_{12}^{2}\right)}\right\}^{-\left(v_{12}+2\right) / 2} d x \\
=\int_{-\infty}^{b_{1}} \frac{\Gamma\left(\left(v_{12}+2\right) / 2\right)}{\Gamma\left(\left(v_{12}+1\right) / 2\right) \sqrt{\pi\left(v_{12}+b_{2}^{2}\right)\left(1-\rho_{12}^{2}\right)}}\left\{1+\frac{\left(x-\rho_{12} b_{2}\right)^{2}}{\left(v_{12}+b_{2}\right)^{2}\left(1-\rho_{12}^{2}\right)}\right\}^{-\left(v_{12}+2\right) / 2} d x .
\end{gathered}
$$

Now, set

$$
\begin{gathered}
v=v_{12}+1, \\
\mu=\rho_{12} b_{2}, \\
\sigma^{2}=\frac{v_{12}+b_{2}^{2}}{v_{12}+1}\left(1-\rho_{12}^{2}\right) .
\end{gathered}
$$

Then

$$
\begin{aligned}
h_{12}\left(u_{1}, u_{2}\right)= & \int_{-\infty}^{b_{1}} \frac{\Gamma\left(\left(v_{12}+2\right) / 2\right)}{\Gamma\left(\left(v_{12}+1\right) / 2\right) \sqrt{\pi\left(v_{12}+b_{2}^{2}\right)\left(1-\rho_{12}^{2}\right)}} \\
& \quad \times\left\{1+\frac{\left(x-\rho_{12} b_{2}\right)^{2}}{\left(v_{12}+b_{2}\right)^{2}\left(1-\rho_{12}^{2}\right)}\right\}^{-\left(v_{12}+2\right) / 2} d x \\
= & \int_{-\infty}^{b_{1}} \frac{\Gamma((v+1) / 2)}{\Gamma(v / 2) \sqrt{\pi v} \sigma}\left\{1+\frac{1}{v} \frac{(x-\mu)^{2}}{\sigma^{2}}\right\}^{-\left(v_{12}+2\right) / 2} \\
= & \int_{-\infty}^{b_{1}} \frac{1}{\sigma} \cdot f_{v}\left(\frac{x-\mu}{\sigma}\right) d x \\
= & \int_{-\infty}^{\left(b_{1}-\mu\right) / \sigma} f_{v}(z) d z \\
= & t_{v}\left(\frac{b_{1}-\mu}{\sigma}\right) .
\end{aligned}
$$


Since

$$
t_{v}(x)=\int_{-\infty}^{x} f_{v}(y) d y
$$

the final expression for $h_{12}\left(u_{1}, u_{2}\right)$ is

$$
h_{12}\left(u_{1}, u_{2}\right)=t_{v_{12}+1}\left(\frac{t_{v_{12}}^{-1}\left(u_{1}\right)-\rho_{12} t_{v_{12}}^{-1}\left(u_{2}\right)}{\sqrt{\left(v_{12}+\left(t_{v_{12}}^{-1}\left(u_{2}\right)\right)^{2}\right)\left(1-\rho_{12}^{2}\right) /\left(v_{12}+1\right)}}\right) .
$$

The inverse function $h_{12}^{-1}$ is given by

$$
h_{12}^{-1}\left(u_{1}, u_{2}\right)=t_{v_{12}}\left\{t_{v_{12}}^{-1}\left(u_{1}\right) \sqrt{\frac{\left(v_{12}+\left(t_{v_{12}}^{-1}\left(u_{2}\right)\right)^{2}\right)\left(1-\rho_{12}^{2}\right)}{v_{12}+1}}+\rho_{12} t_{v_{12}}^{-1}\left(u_{2}\right)\right\} .
$$

Using the simplified pair-copulae functions $h_{12}\left(u_{1}, u_{2}\right)$ and $h_{12}^{-1}\left(u_{1}, u_{2}\right)$ shown previously, the general algorithm for sampling $\left(x_{1}, x_{2}, x_{3}, \ldots, x_{N}\right)$ from the "theoretical" Student's copula becomes.

(1) Sample $w_{i}$ where $i=1,2, \ldots, m$ independent standard uniform.

(2) Given that $F_{1 \mid 2}\left(u_{2} \mid u_{1}\right)=h_{12}\left(u_{1}, u_{2}\right)$ and $F_{1 \mid 2}^{-1}\left(u_{2} \mid u_{1}\right)=h_{12}^{-1}\left(u_{1}, u_{2}\right)$, we can calculate $x_{1}, x_{2}, x_{3}, \ldots, x_{m}$ as follows:

$$
\begin{aligned}
& x_{1}=w_{1}, \\
& x_{2}=F_{2 \mid 1}^{-1}\left(w_{2} \mid x_{1}\right)=h_{12}^{-1}\left(w_{2}, x_{1}\right), \\
& x_{3}=F_{3 \mid 1,2}^{-1}\left(w_{3} \mid x_{1}, x_{2}\right)=h^{-1}\left(h^{-1}\left(w_{3}, h\left(x_{2}, x_{1}\right)\right), x_{1}\right), \\
& \quad \vdots \\
& x_{m}=F_{N \mid 1,2, \ldots, N-1}^{-1}\left(w_{N} \mid x_{1}, x_{2}, \ldots, x_{N-1}\right), \\
& =h^{-1}\left(\left(\cdots h^{-1}\left(h^{-1}\left(w_{N-1},\left(h^{-1}\left(w_{N}, h\left(x_{N-1}, x_{1}\right)\right)\right), x_{1}\right)\right) \cdots\right), x_{1}\right),
\end{aligned}
$$




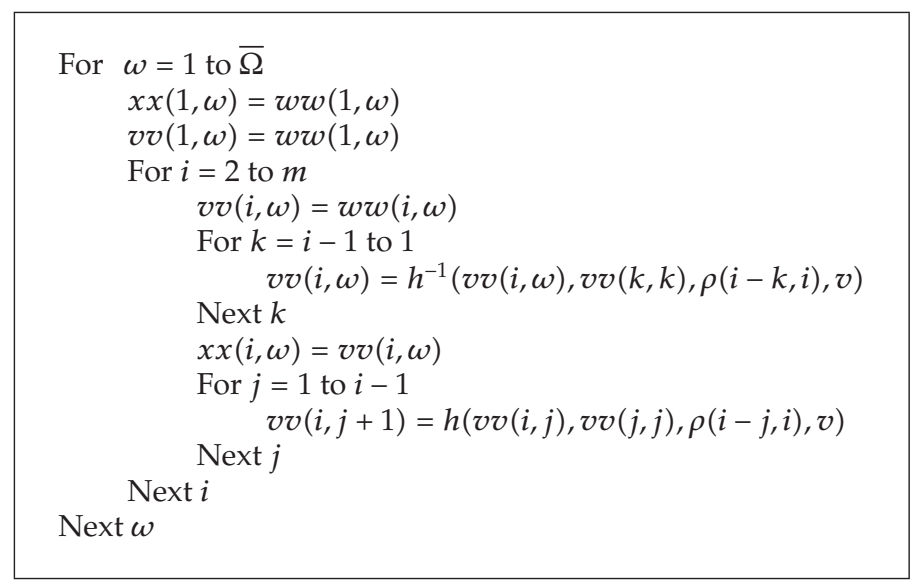

Algorithm 1

where

$$
\begin{aligned}
& F\left(x_{2} \mid x_{1}\right)=h\left(x_{2}, x_{1}\right), \\
& F\left(x_{3} \mid x_{1}, x_{2}\right)=h\left(F\left(x_{3} \mid x_{1}\right), F\left(x_{2} \mid x_{1}\right)\right), \\
&=h\left(h\left(x_{3}, x_{1}\right), h\left(x_{2}, x_{1}\right)\right), \\
& F\left(x_{4} \mid x_{1}, x_{2}, x_{3}\right)=h\left(F\left(x_{4} \mid x_{1}, x_{2}\right), F\left(x_{3} \mid x_{1}, x_{2}\right)\right), \\
&=h\left(h\left(h\left(x_{4}, x_{1}\right), h\left(x_{2}, x_{1}\right)\right), h\left(h\left(x_{3}, x_{1}\right), h\left(x_{2}, x_{1}\right)\right)\right), \\
& \vdots
\end{aligned}
$$

\section{B. Monte Carlo Simulation Algorithm for the "Full" Network}

As before, let $m$ be the total number of firms in the network and let $\bar{\Omega}$ be the total number of revenue scenarios for each firm in the network. $h$ and $h^{-1}$ are the functions derived previously. The detailed algorithm of our simulation is as in Algorithm 1.

\section{Acknowledgment}

The authors thank Fitch Ratings for their financial and intellectual support. All errors are ours.

\section{Endnotes}

1. By this, we mean that the dependence between tail events is stronger for the $t$-copula than for the Gaussian copula. 


\section{References}

[1] U. Cherubini, E. Luciano, and W. Vecchiato, Copula Methods in Finance, Wiley Finance Series, John Wiley \& Sons, Chichester, UK, 2004.

[2] P. Embrechts, F. Lindskog, and A. McNeil, "Modelling dependence with copulas and applications to risk management," in Handbook of Heavy Tailed Distributions in Finance, S. Rachev, Ed., chapter 8, pp. 329-384, Elsevier, Amsterdam, The Netherlands, 2003.

[3] H. Joe, Multivariate Models and Dependence Concepts, vol. 73 of Monographs on Statistics and Applied Probability, Chapman \& Hall, London, UK, 1997.

[4] R. B. Nelsen, An Introduction to Copulas, Springer Series in Statistics, Springer, New York, NY, USA, 2nd edition, 2007.

[5] D. Cossin and H. Schellhorn, "Credit risk in a network economy," Management Science, vol. 53, no. 10, pp. 1604-1617, 2007.

[6] C. Bluhm, "CDO Modeling: Techniques, Examples, and Applications," 2003.

[7] H. Leland, "Corporate debt value, bond covenants, and optimal capital structure," Journal of Finance, vol. 49, no. 4, pp. 1213-1252, 1994.

[8] H. Leland and K. Toft, "Optimal capital structure, endogenous bankruptcy, and the term structure of credit spreads," Journal of Finance, vol. 51, no. 3, pp. 987-1019, 1996.

[9] D. Lando, Credit Risk Modelling, Princeton University Press, Princeton, NJ, USA, 2004.

[10] Y. Malevergne and D. Sornette, "Testing the Gaussian copula hypothesis for financial assets dependences," Quantitative Finance, vol. 3, no. 4, pp. 231-250, 2003.

[11] K. Aas, C. Czado, A. Frigessi, and H. Bakken, "Pair-Copula Constructions of Multiple Dependence," Institut Fur Statistik Sonderforschungsbereich 386, Paper 487, 2006.

[12] F. Massey, "The Kolmogorv-Smirnov test for goodness of fit," Journal of the American Statistical Association, vol. 46, no. 253, pp. 68-78, 1951.

[13] H. Milbrodt and H. Strasser, "On the asymptotic power of the two-sided Kolmogorov-Smirnov test," Journal of Statistical Planning and Inference, vol. 26, no. 1, pp. 1-23, 1990. 


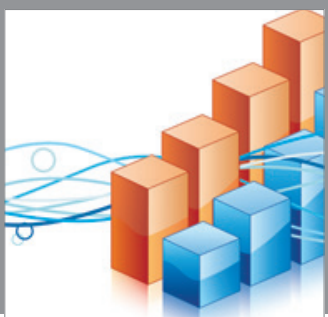

Advances in

Operations Research

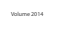

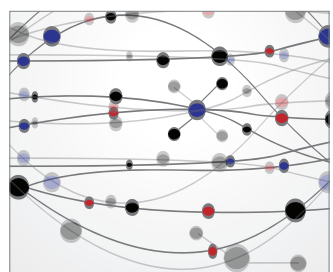

\section{The Scientific} World Journal
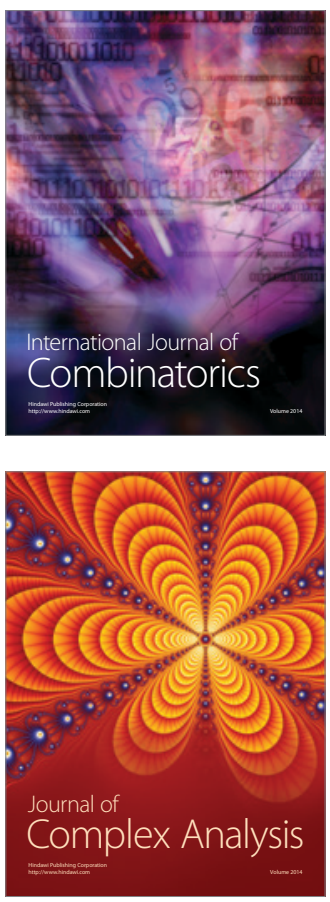

International Journal of

Mathematics and

Mathematical

Sciences
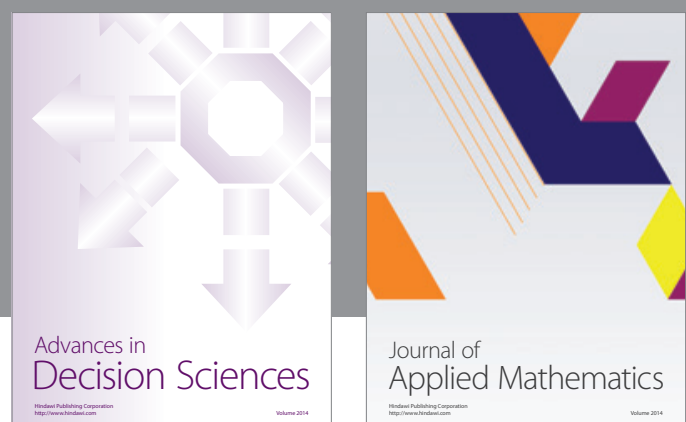

Journal of

Applied Mathematics
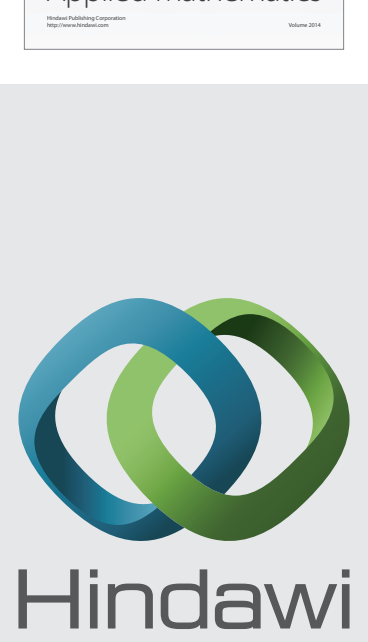

Submit your manuscripts at http://www.hindawi.com
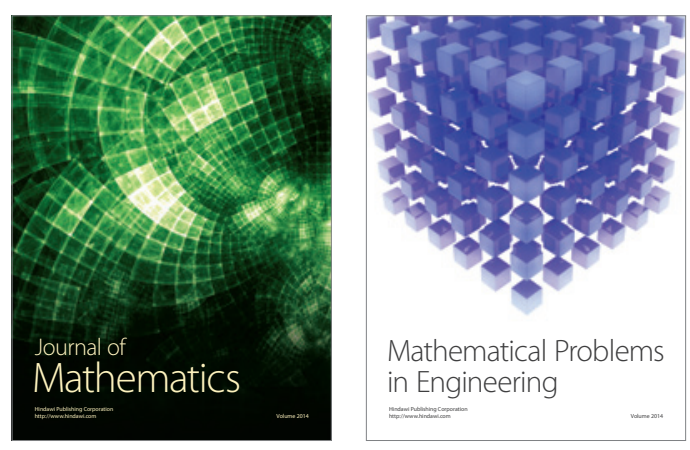

Mathematical Problems in Engineering
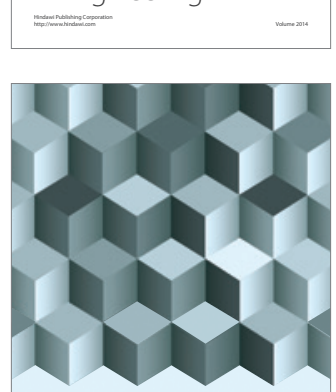

Journal of

Function Spaces
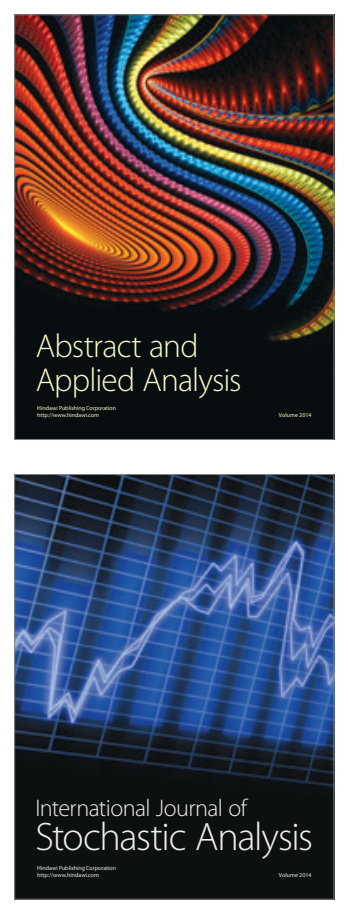

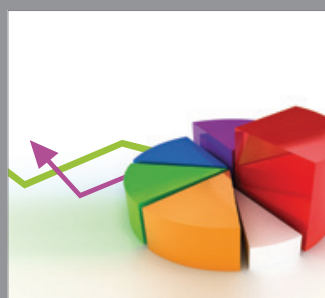

ournal of

Probability and Statistics

Promensencen
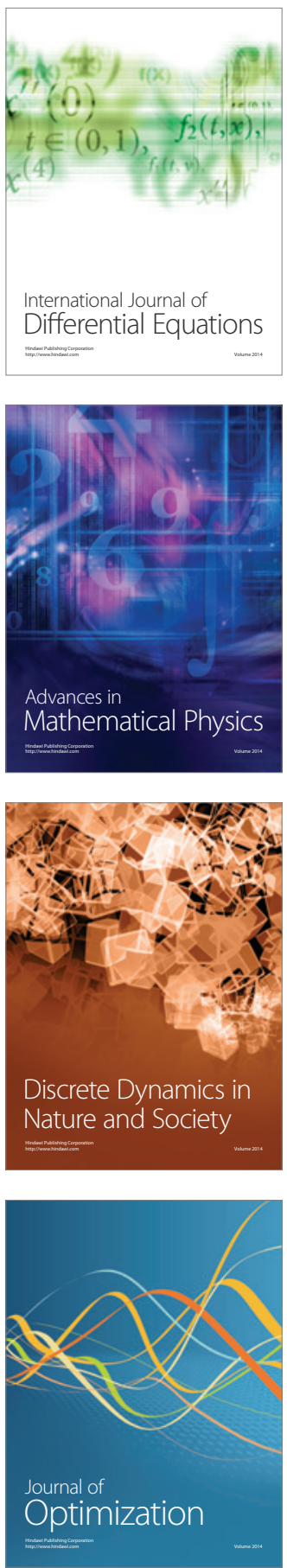